Multicenter study of intravenous recombinant tissue plasminogen activator infusion around Hiroshima, Japan -HARP Registry Study-

\author{
Shiro Aoki ${ }^{11}$, Naohisa Hosomi ${ }^{1}$, Yoshimasa Sueda ${ }^{1,2)}$, Tomoyuki Kono ${ }^{1}$, \\ Kazuhiro Takamatsu ${ }^{3)}$, Hideo Ohyama ${ }^{4)}$, Tsuyoshi Torii'2), \\ Takeshi Kitamura ${ }^{5)}$, Eiichi Nomura ${ }^{6}$, Koichi Noda ${ }^{7)}$, Toshiho Ohtsuki ${ }^{1}$, \\ Masayasu Matsumoto1), \\ for the HARP Registry Study Group
}

1. Department of Clinical Neuroscience and Therapeutics, Hiroshima University Graduate School of Biomedical and Health Sciences, Hiroshima, Japan

2. Department of Neurology, National Hospital Organization Kure Medical Center, Kure, Japan

3. Department of Neurology, Brain Attack Center Ota Memorial Hospital, Fukuyama, Japan

4. Department of Cardiology, Osaka Neurosurgical Hospital, Kagawa, Japan

5. Department of Neurology, Chugoku Rosai Hospital, Kure, Japan

6. Department of Neurology, Suiseikai Kajikawa Hospital, Hiroshima, Japan 
7. Department of Neurology, National Hospital Organization Higashihiroshima Medical Center, Higashihiroshima, Japan

\section{Sources of Funding}

This study was supported in part by research grants from JSPS KAKENHI Grant Number (23590598, 15K08615), the Japan Science and Technology Agency (AS242Z02592P), the Smoking Research Foundation, the Tsuchiya Foundation, and the Japan Heart Foundation.

\section{Disclosures}

Dr. Hosomi reports Honoraria from Mochida Pharmaceutical CO., LTD. outside the submitted work. The other authors report no conflicts of interest.

\section{Address correspondence to:}

Naohisa Hosomi, MD, PhD, FAHA

Department of Clinical Neuroscience and Therapeutics, Hiroshima University Graduate School of Biomedical and Health Sciences

1-2-3 Kasumi, Minami-ku, Hiroshima 734-8551, Japan

Fax: +81-82-505-0490. Tel: +81-82-257-5201

E-mail: nhosomi@hiroshima-u.ac.jp

Running title: Multicenter study of IV rtPA around Hiroshima 


\section{Abstract}

Objective: Approximately ten years have passed since intravenous (IV) recombinant tissue plasminogen activator (rtPA) therapy was approved in Japan. The aim of this retrospective study was to identify the effectiveness and safety of IV alteplase therapy with the Japanese original dose around Hiroshima via consideration of the patients' backgrounds, examination findings, and outcomes. Methods: All consecutive ischemic stroke patients who received IV alteplase therapy between October 2005 and October 2010 were registered, and statistical analysis was performed. Results: Four hundred twenty-nine ischemic stroke patients (172 female [40.1\%], mean age $73.7 \pm 11.8$ years) were registered. The proportion of patients over 75 years old was 51.5\% (221 patients). The median NIH Stroke Scale (NIHSS) scores at admission were 13 (interquartile range [IQR], 9-19), and the NIHSS scores 24 hours after alteplase infusion were 8 (IQR, 3-15). The proportion of intracerebral hemorrhage within the initial 36 hours was $20.2 \%$ (86 patients). After the multivariate regression analysis, a previous history of hypertension (Odds Ratio [OR] 4.14, 95\% confidence interval [CI] 1.32-14.79, $\mathrm{p}=0.01)$ and no recanalization (OR 10.10, 95\%CI 3.03-39.33, $\mathrm{p}<0.0001)$ were independently associated with a modified Rankin Scale (m-RS) score $\geq 2$ at 3 months. Patients over 75 years old were not significantly associated to an intracerebral hemorrhage within the initial 36 hours and an $\mathrm{m}-\mathrm{RS}$ score $\geq 2$ at 3 months. Conclusions: The results of our study demonstrated that IV alteplase therapy with the Japanese original dose was effective and 
exhibited a safety profile similar to other studies. We should not hesitate to IV alteplase therapy simply because of advanced age. 


\section{Introduction}

Approximately ten years have passed since intravenous (IV) recombinant tissue plasminogen activator (rtPA) therapy was approved in Japan. The efficacy and safety of the Japanese original low-dose $(0.6 \mathrm{mg} / \mathrm{kg}) \mathrm{IV}$ alteplase have been demonstrated in previous studies.1,2 In addition, the administration time of alteplase has been increased to within 4.5 hours of stroke onset since August 31 2012 in Japan; thus, an increase in alteplase therapy is also expected.

However, limitations of IV alteplase therapy have been identified especially in cases that involve occlusion of the internal carotid artery or proximal middle cerebral artery. ${ }^{3,4}$ Mechanical thrombectomy was first performed in 2010, and it has been effective in cases refractory to IV alteplase therapy. ${ }^{5-7}$

To perform high-quality stroke therapy under conditions in which the treatment approach dynamically changes, the continued review of large clinical case series is necessary. Furthermore, because Japan has still been advancing its super-aging society, it is important to clarify the characteristics of these cases in difference in age. The risk of IV alteplase therapy for elderly patients remains controversial.

Thus, we performed a multicenter study (Hiroshima Acute stroke Retrospective and Prospective [HARP] Registry Study) to clarify the characteristics of acute stroke patients around Hiroshima, Japan. The aim of this retrospective study was to identify the effectiveness and safety of IV alteplase therapy with the Japanese original dose around Hiroshima via 
consideration of the patients' backgrounds, examination findings, and outcomes.

\section{Patients and Methods}

The HARP Study Group was composed of 7 Japanese stroke centers located around Hiroshima, Japan. All consecutive ischemic stroke patients who received IV alteplase therapy between October 2005 and October 2010 were registered. Patient eligibility for alteplase therapy was primarily determined based on the Japanese guidelines for IV alteplase therapy, which follow the inclusion and exclusion criteria used in the National Institute of Neurological Disorders and Stroke (NINDS) study $^{8}$ and Japan Alteplase Clinical Trial (J-ACT). ${ }^{9}$

Each patient received a single alteplase dose of $0.6 \mathrm{mg} / \mathrm{kg}$ IV with $10 \%$ administered as a bolus within 3 hours of stroke onset, followed by a continuous IV infusion of the remaining drug over 1 hour. The use of an antithrombotic agent was prohibited for 24 hours after stroke onset, blood pressure was maintained at $<180 / 105 \mathrm{mmHg}$, and neurological signs and symptoms were frequently monitored. Prior to alteplase therapy, all patients underwent computed tomography (CT) or magnetic resonance imaging (MRI). The stroke subtype was determined based on CT or MRI findings, electrocardiography, and carotid artery and cardiac ultrasound findings by at least two stroke specialists according to the Trial of Org 10172 in Acute Stroke Treatment (TOAST) classification. ${ }^{10}$ Recanalization was assessed by 
the MR angiography 24 hours after alteplase infusion and was graded as complete, partial, or no recanalization as follows: (1) complete recanalization, which indicated reappearance of the entire occluded artery and the distal branch of the vessels; (2) partial recanalization, which indicated restoration of part of the distal vessel supplied by the occluded artery; and (3) no recanalization, which indicated persistent occlusion. Recanalization was defined as complete or partial recanalization. Intracerebral hemorrhage (ICH) was defined as CT evidence of new ICH within the initial 36 hours. White matter lesions (WMLs) were graded as the degree of severity of the WMLs via visual inspection using the Fazekas classification of WMLs as follows: none (grade 0), punctate (grade 1), early confluent (grade 2), and confluent (grade 3) lesions. ${ }^{11}$ The neurological severity was evaluated using the National Institutes of Health Stroke Scale (NIHSS) at admission and 24 hours after alteplase infusion. ${ }^{12}$ The patients' clinical outcomes were evaluated with the modified Rankin scale (m-RS) at discharge and 3 months after stroke onset. ${ }^{13} \mathrm{~A}$ favorable outcome was defined as an m-RS score of 0 1 at 3 months after stroke onset. The study was approved by the Institutional Review Board (IRB) of each institute.

Hypertension was defined as the use of anti-hypertensive medicines prior to admission or a confirmed blood pressure $\geq 140 / 90 \mathrm{mmHg}$ at rest 2 weeks after stroke onset. Diabetes mellitus was defined as an $\mathrm{HbA} 1 \mathrm{c}$ of $\geq 6.5 \%$, a fasting blood sugar $\geq 126 \mathrm{mg} / \mathrm{dl}$, or the use of anti-diabetic medicines. Hyperlipidemia was defined as a total cholesterol (T-chol) $\geq 220 \mathrm{mg} / \mathrm{dl}$, a 
low-density lipoprotein cholesterol (LDL-chol) $\geq 140 \mathrm{mg} / \mathrm{dl}$ at admission, or the use of anti-hyperlipidemia medications. Atrial fibrillation was diagnosed based on electrocardiographic findings at admission or during hospitalization or a previous history of atrial fibrillation.

Statistical analysis was performed using JMP 11.2 statistical software (SAS Institute, Inc., Cary, North Carolina, USA). The data are presented as the mean $\pm \mathrm{SD}$ or the median (minimum-maximum) for continuous variables. Statistical analyses for the comparisons of the two groups were performed using Student's t-test or the Mann-Whitney U test for continuous variables and the chi-squared test or Fisher's exact test for categorical variables. P-values $<0.05$ were considered statistically significant. Multivariate analyses were performed using a stepwise analysis to determine the predictors of an ICH within the initial 36 hours, and an $\mathrm{m}-\mathrm{RS}$ score $\leq 1$ at 3 months was based on the characteristics detailed in Tables 1 and 2. For each outcome, a backward selection procedure was performed using $p>0.10$ of the likelihood ratio test for exclusion.

\section{Results}

Four hundred twenty-nine ischemic stroke patients (172 female [40.1\%], mean age $73.7 \pm 11.8$ years) were registered. Table 1 shows the baseline characteristics of all registered patients. There were 285 patients with hypertension $(66.4 \%)$ and 220 patients with atrial fibrillation (51.3\%). The leading stroke subtype was cardioembolism (59.4\%) followed by large artery 
atheroscrelosis (24.0\%). The median time between stroke onset and alteplase infusion was 135 minutes (interquartile range [IQR], 110-158). The median NIHSS scores at admission were 13 (IQR, 9-19), and the NIHSS scores 24 hours after alteplase infusion were 8 (IQR, 3-15).

The proportion of patients over 75 years old was $51.5 \%$ (221 patients). The proportion of female was significantly higher in patients over 75 years old than patients under 75 years old $(\mathrm{p}<0.0001)$. Patients over 75 years more commonly had hypertension $(\mathrm{p}=0.02)$, atrial fibrillation $(\mathrm{p}<0.0001)$, and previous history of stroke $(\mathrm{p}=0.0004)$ than those under 75 years old. NIHSS scores at admission were significantly higher in patients over 75 years old than those under 75 years old $(\mathrm{p}<0.0001)$.

Table 2 shows the imaging findings. In $355(82.8 \%)$ out of 429 patients, the location of the occluded artery was identified. The leading site of the arterial occlusion was the middle cerebral artery $(76.0 \%)$ followed by the internal carotid artery (13.5\%). The recanalization of the occluded artery was assessed in 260 patients. One hundred fifty-seven patients exhibited complete or partial recanalization (60.4\%). The proportion of ICH within the initial 36 hours was $20.2 \%$ (86 patients). Three hundred fifty-eight patients had MRI findings consistent with WMLs; 115 patients were grade 0 (32.1\%), 140 patients were grade $1(39.1 \%), 66$ patients were grade $2(18.4 \%)$, and 37 patients were grade $3(10.3 \%)$. The severity of WMLs is higher in patients over 75 years old than in patients under 75 years old.

Based on the multivariate regression analysis using a backward selection 
method, a previous history of atrial fibrillation (Odds Ratio (OR) 5.70, 95\% confidence interval (CI) $1.93-20.44, \mathrm{p}=0.001$ ), the NIHSS score at admission (OR 1.06, 95\%CI 1.01-1.11, p=0.03), and recanalization (OR 4.95, 95\%CI 2.23-12.29, $\mathrm{p}<0.0001$ ) were independently associated with ICH within the initial 36 hours (Table 3). The functional outcomes (m-RS) at 3 months were evaluated in 224 patients. One hundred patients (44.6\%) had an m-RS score $\leq 1$ at 3 months. When 33 patients with a premorbid m-RS score $\geq 2$ were excluded from the analysis, $99(51.9 \%)$ of 191 patients had an m-RS score $\leq 1$ (Figure 1). The proportion of patients with an $\mathrm{m}-\mathrm{RS}$ score $\leq 1$ at 3 months was significantly lower in patients over 75 years old than patients under 75 years old $(\mathrm{p}<0.0001)$. After the multivariate regression analysis using a backward selection method, a previous history of hypertension (OR 4.14, 95\%CI 1.3214.79, $\mathrm{p}=0.01$ ) and no recanalization (OR 10.10, 95\%CI 3.03-39.33, $\mathrm{p}<0.0001$ ) were independently associated with an $\mathrm{m}-\mathrm{RS}$ score $\geq 2$ at 3 months (Table 4).

\section{Discussion}

Our study identified the characteristics of patients who received IV rtPA therapy around Hiroshima, Japan. The leading site of arterial occlusion was the middle cerebral artery (76.0\%) followed by the internal carotid artery (13.5\%). The proportion of ICH within the initial 36 hours was $20.2 \%$, whereas an m-RS score $\leq 1$ at 3 months was $44.6 \%$. These results were similar to previous reports that demonstrated the efficacy and safety of the 
Japanese original low-dose $(0.6 \mathrm{mg} / \mathrm{kg})$ IV alteplase. ${ }^{1,2}$

In this study, the mean age of the patients was $73.7 \pm 11.8$ years, and the proportion of females was $40.1 \%$. The previous Japanese low-dose (0.6 $\mathrm{mg} / \mathrm{kg}$ ) alteplase studies, the Japan post-Marketing Alteplase Registration Study (J-MARS) ${ }^{1}$ and the Stroke Acute Management with Urgent Risk-factor Assessment and Improvement (SAMURAI) Study ${ }^{2}$ all demonstrated similar patient characteristics (J-MARS, 72 years, female $37.9 \%$; SAMURAI, $72 \pm 12$ years, female $37.2 \%$ ). The proportions of the risk factors are also similar in these 3 studies. Therefore, we assume that the results of our study may reflect the characteristics of general Japanese ischemic stroke patients with IV alteplase treatment.

Focusing on the difference in age, the proportion of female, hypertension, atrial fibrillation, and previous history of stroke was significantly higher in patients over 75 years old than patients under 75 years old in this study. The outcomes of patients over 75 years old were poorer than those of patients 75 years old. These results are thought to be similar to previous alteplase studies.

Our study demonstrates that an ICH within the initial 36 hours is independently associated with a previous history of atrial fibrillation, the NIHSS score at admission, and recanalization with IV alteplase. Previous studies have reported that the predisposing ICH factors after thrombolysis included renal dysfunction, ${ }^{14}$ atrial fibrillation, ${ }^{14}$ post-thrombolysis blood pressure, ${ }^{15}$ and large baseline diffusion-weighted imaging (DWI) volumes. ${ }^{15}$ 
In this study, we did not evaluate the renal function or baseline DWI. However, the baseline DWI volumes were significantly associated with the NIHSS score at admission in the SAMURAI study. ${ }^{16}$ Thus, a high NIHSS score at admission may reflect large baseline DWI volumes in our study. Additionally, atrial fibrillation is strongly associated with cardioembolism, and cardioembolism is a predictive factor of early hemorrhagic transformation in acute ischemic stroke. ${ }^{17} \mathrm{~A}$ high NIHSS score at admission and recanalization are also related to cardioembolism, which may be the reason that these factors are independently associated with an ICH within the initial 36 hours.

The clinical outcome is an important endpoint after thrombolysis. Our study demonstrates that a previous history of hypertension and no recanalization are independently associated with an $\mathrm{m}-\mathrm{RS}$ score $>2$ at 3 months. The SAMURAI study demonstrated that age, the NIHSS score at admission, glucose levels, internal carotid artery occlusion, ASPECTS on CT, and IV anti-hypertensive medications immediately prior to rtPA were associated with a m-RS score $>2$ at 3 months. ${ }^{2}$ Additionally, J-ACT II demonstrated that no recanalization of the occluded artery by 24 hours after rtPA infusion was strongly associated with a poor outcome (OR 12.5, 95\%CI 2.5-62.4, $\mathrm{p}<0.001) .{ }^{3}$ We suggest the results of our study are similar to these Japanese rtPA studies and reflect the actual acute ischemic stroke clinical practice in Japan.

Patients over 75 years old were not significantly associated to an ICH within 
the initial 36 hours and an m-RS score $>2$ at 3 months in this study. Elderly patients who receive IV alteplase therapy were generically thought to be likely to occur with hemorrhagic transformation and have poor outcomes. However, a growing body of evidence in recent years is accumulating to demonstrate that IV alteplase therapy for elderly patients is relatively safe. 18,19 Our results surely demonstrated that patients over 75 years old had poorer outcome than patients under 75 years old, but the proportion of good outcome was no different from other aleplase studies of all ages. Therefore, we should not hesitate to IV alteplase therapy simply because of advanced age.

This study has some limitations. First, because these are retrospectively collected data, some imaging findings and m-RS scores at 3 months are missing. In our study, only $44 \%$ of patients were obtained their clinical outcome (m-RS scores at 3 months). It may influence on our slight favorable result (m-RS 0-1,51\%) in comparison previous studies. Analyzing those patients with the data of outcome, they were relatively younger $(70.5 \pm 11.8$ years) and lower NIHSS score at admission (11 [8-18]) than the patients in the previous Japanese alteplase studies (SAMURAI; 72 \pm 12 years, 13 [7.3-19], J-MARS; 72 years, 15 [9-20]). We consider that those results may also influence on the favorable outcome of our study compared with the previous studies. Other backgrounds and imaging findings are almost similar to the previous studies. Second, because all patients in this study live around Hiroshima, Japan, we cannot exclude the possibility that a regional bias 
exists. However, most of our results are similar to previous Japanese rtPA trials. Therefore, we assume that these limitations do not have a substantial influence on our results.

In conclusion, the results of the HARP Registry Study demonstrated that IV rtPA therapy with the Japanese original dose was effective and exhibited a safety profile similar to other studies. We should not hesitate to IV alteplase therapy simply because of advanced age. Further prospective studies are necessary to clarify the characteristics of the cases in each region.

\section{Acknowledgements}

We would like to show our appreciation to the participated HARP study collaborators. 


\section{References}

1. Nakagawara J, Minematsu K, Okada Y, et al. for J-MARS Investigators. Thrombolysis With $0.6 \mathrm{mg} / \mathrm{kg}$ Intravenous Alteplase for Acute Ischemic Stroke in Routine Clinical Practice: The Japan post-Marketing Alteplase Registration Study (J-MARS). Stroke 2010; 41: 1984-1989.

2. Toyoda K, Koga M, Naganuma M, et al. Stroke Acute Management with Urgent Risk-factor Assessment and Improvement Study Investigators. Routine use of intravenous low-dose recombinant tissue plasminogen activator in Japanese patients: general outcomes and prognostic factors from the SAMURAI register. Stroke 2009; 40: 3591-3595.

3. Mori E, Minematsu K, Nakagawara J, et al. Japan Alteplase Clinical Trial II Group. Effects of $0.6 \mathrm{mg} / \mathrm{kg}$ intravenous alteplase on vascular and clinical outcomes in middle cerebral artery occlusion: Japan Alteplase Clinical Trial II (J-ACT II). Stroke 2010; 41: 461-465.

4. Bhatia R, Hill MD, Shobha $\mathrm{N}$, et al. Low rates of acute recanalization with intravenous recombinant tissue plasminogen activator in ischemic stroke: real-world experience and a call for action. Stroke 2010; 41: 2254-2258.

5. Saver JL, Jahan R, Levy EI, et al. SWIFT Trialists. Solitaire flow restoration device versus the Merci Retriever in patients with acute ischaemic stroke (SWIFT): a randomised, parallel-group, non-inferiority trial. Lancet 2012; 380: 1241-1249.

6. Nogueira RG, Lutsep HL, Gupta R, et al. TREVO 2 Trialists. Trevo versus 
Merci retrievers for thrombectomy revascularisation of large vessel occlusions in acute ischaemic stroke (TREVO 2): a randomised trial. Lancet 2012; 380: 1231-1240.

7. Berkhemer OA, Fransen PS, Beumer D, et al. MR CLEAN Investigators. A randomized trial of intraarterial treatment for acute ischemic stroke. $\mathrm{N}$ Engl J Med 2015; 372: 11-20.

8. The National Institute of Neurological Disorders and Stroke rt-PA Stroke Study Group. Tissue plasminogen activator for acute ischemic stroke. N Engl J Med 1995; 333: 1581-1587.

9. Yamaguchi T, Mori E, Minematsu K, et al. Japan Alteplase Clinical Trial (J-ACT) Group. Alteplase at $0.6 \mathrm{mg} / \mathrm{kg}$ for acute ischemic stroke within 3 hours of onset: Japan Alteplase Clinical Trial (J-ACT). Stroke 2006; 37: 1810-1815.

10.Adams HP, Jr., Bendixen BH, Kappelle LJ, et al. Classification of subtype of acute ischemic stroke. Definitions for use in a multicenter clinical trial. TOAST. Trial of Org 10172 in Acute Stroke Treatment. Stroke 1993; 24: $35-41$.

11. Fazekas F, Chawluk JB, Alavi A, et al. MR signal abnormalities at $1.5 \mathrm{~T}$ in Alzheimer's dementia and normal aging. AJR Am J Roentgenol 1987; 149: $351-356$.

12. Brott T, Adams HP, Jr., Olinger CP, et al. Measurements of acute cerebral infarction: a clinical examination scale. Stroke 1989; 20: 864-870.

13.van Swieten JC, Koudstaal PJ, Visser MC, et al. Interobserver agreement 
for the assessment of handicap in stroke patients. Stroke 1988; 19: 604-607.

14. Naganuma M, Koga M, Shiokawa Y, et al. Reduced estimated glomerular filtration rate is associated with stroke outcome after intravenous rt-PA: the Stroke Acute Management with Urgent Risk-Factor Assessment and Improvement (SAMURAI) rt-PA registry. Cerebrovasc Dis 2011; 31: 123-129.

15. Butcher K, Christensen S, Parsons M, et al. EPITHET Investigators. Postthrombolysis blood pressure elevation is associated with hemorrhagic transformation. Stroke 2010; 41: 72-77.

16. Nezu T, Koga M, Kimura K, et al. Pretreatment ASPECTS on DWI predicts 3-month outcome following rt-PA: SAMURAI rt-PA Registry. Neurology 2010; 75: 555-561.

17. Paciaroni M, Agnelli G, Corea F, et al. Early hemorrhagic transformation of brain infarction: rate, predictive factors, and influence on clinical outcome: results of a prospective multicenter study. Stroke 2008; 39: 2249-2256.

18. Bhatnagar P, Sinha D, Parker RA, et al. Intravenous thrombolysis in acute ischaemic stroke: a systematic review and meta-analysis to aid decision making in patients over 80 years of age. J Neurol Neurosurg Psychiatry 2011; 82: 712-717.

19. Koga M, Shiokawa Y, Nakagawara J, et al. Low-dose intravenous recombinant tissue-type plasminogen activator therapy for patients with 
stroke outside European indications: Stroke Acute Management with Urgent Risk-factor Assessment and Improvement (SAMURAI) rtPA Registry. Stroke 2012; 43: 253-255.

\section{Figure legends}

Figure 1. m-RS score at 3 months in the all patients, patients over 75 years old, and patients under 75 years old with premorbid m-RS 0-1. 


\section{Appendix: Participating HARP Registry Study Institutions}

Brain attack Center Ota Memorial Hospital (Kazuhiro Takamatsu, Taisei

Ota, Isao Inoue, Shihori Kitae, Atsushi Tsuchiya, Nobutoshi Morimoto,

Shoko Nagotani, Yasuyuki Ota, Motonori Takamiya, Hayato Matsushima,

Muneyuki Makawa, Yoshio Omote, Hiroki Fujii, Miyuki Okamoto, Satoshi

Kubo, Kota Sato, Taijyun Yunoki, and Masaru Kuriyama), Osaka

Neurosurgical Hospital (Hideo Ooyama, Yuzo Kuroda, Keita Moriwaki,

Naofumi Isono, Taro Yamashita, Naoki Hayashi, Shigeki Sadahiro, and

Naohiro Osaka), National Hospital Organization Kure Medical Center

(Tsuyoshi Torii, Eiichi Ohnuki, Tomokazu Nishikawa, Ryo Shimomura,

Yukari Shinozaki, Syuichiro Neshige, and Atsuo Yamada), Chugoku Rosai

Hospital (Takeshi Kitamura, Hiromasa Toji, Hideo Terasawa, Shinya

Yamaguchi, and Satoshi Kataoka), Suiseikai Kajikawa Hospital (Eiichi

Nomura, Yasuyo Mimori, Yumiko Kaseda, Junko Ikeda, Hiromitsu Naka,

Satomi Kushitani, Eiji Imamura, Juri Kitamura, and Shinichi Wakabayashi),

National Hospital Organization Higashihiroshima Medical Center (Koichi

Noda, Kazue Tajima, Tomohito Sugiura, Yoshio Murakami, Takeo Shishido, 
and Yoshito Nagano), and Hiroshima University Hospital (Takemori

Yamawaki, Hideshi Kawakami, Sadao Katayama, Toshiho Ohtsuki, Hiroshi

Yamashita, Hirofumi Maruyama, Takafumi Miyachi, Tetsuya Takahashi,

Takeshi Nakamura, Tomohiko Ohshita, Kazuhide Ochi, Masahiro Higaki,

Naoki Matsuoka, Daisuke Matsuura, Nasanori Hiji, Hiroki Ueno, Keita

Kondo, Hiromasa Fukuba, Yoshimasa Sueda, Yu Yamazaki, Michie, Miyoshi,

Kayoko Ishihara, Aiko Ishihara, Tomoyuki Kono, Shiro Aoki, Takashi

Kurashige, Tomoya Mukai, Teppei Kotozaki, Akiko Segawa, Ikuko Takeda,

Masahiro Nakamori, Tomohisa Nezu, Eisuke Dohi, Takamichi Sugimoto,

Yuki Hayashi, Mayu Sekine, Michihiro Kono, Naoyuki Hara, and Masayasu

Matsumoto). 
Table 1. Baseline Characteristics of the Patients

\begin{tabular}{|c|c|c|c|c|}
\hline Factors & $\begin{array}{l}\text { All patients } \\
\quad(\mathrm{n}=429)\end{array}$ & $\begin{array}{l}\text { Patients } \\
\text { over } 75 \\
\text { years old } \\
(\mathrm{n}=221)\end{array}$ & $\begin{array}{l}\text { Patients } \\
\text { under } 75 \\
\text { years old } \\
(\mathrm{n}=208)\end{array}$ & $p$-value \\
\hline Age, years & $73.7 \pm 11.8$ & $82.5 \pm 5.2$ & $64.4 \pm 9.5$ & $<0.0001$ \\
\hline $\begin{array}{l}\text { Gender, female } \\
\text { Risk factors }\end{array}$ & $172(40.1 \%)$ & $118(53.4 \%)$ & $54(26.0 \%)$ & $<0.0001$ \\
\hline Hypertension & $289(66.4 \%)$ & $160(72.4 \%)$ & $129(62.0 \%)$ & 0.02 \\
\hline Diabetes mellitus & $80(18.6 \%)$ & $44(19.9 \%)$ & $36(17.3 \%)$ & 0.49 \\
\hline Hyperlipidemia & $128(29.8 \%)$ & $60(27.2 \%)$ & $68(32.7 \%)$ & 0.21 \\
\hline Atrial fibrillation & $220(51.3 \%)$ & $135(61.1 \%)$ & $85(40.9 \%)$ & $<0.0001$ \\
\hline Previous stroke & $93(21.8 \%)$ & $63(28.5 \%)$ & $30(14.6 \%)$ & 0.0004 \\
\hline $\begin{array}{l}\text { Prestroke independence, } \\
\text { m-RS 0-1 }\end{array}$ & $381(88.8 \%)$ & $182(82.4 \%)$ & $199(95.7 \%)$ & $<0.0001$ \\
\hline $\begin{array}{l}\text { Prior use of } \\
\text { anticoagulants }\end{array}$ & $57(13.3 \%)$ & $35(15.8 \%)$ & $22(10.6 \%)$ & 0.02 \\
\hline $\begin{array}{l}\text { Prior use of antiplatelet } \\
\text { medication }\end{array}$ & $108(25.2 \%)$ & $70(31.7 \%)$ & $38(18.3 \%)$ & 0.002 \\
\hline $\begin{array}{l}\text { Prior use of } \\
\text { antihypertensives }\end{array}$ & $228(53.1 \%)$ & $134(60.6 \%)$ & $94(45.2 \%)$ & 0.001 \\
\hline Prior use of statins & $81(18.9 \%)$ & $46(20.8 \%)$ & $35(16.8 \%)$ & 0.29 \\
\hline
\end{tabular}


Stroke subtype

0.0005

Cardioembolism

$255(59.4 \%) \quad 152(68.8 \%) \quad 103(49.5 \%)$

Large artery

$103(24.0 \%) \quad 46(20.8 \%) \quad 57(27.4 \%)$

atheroscrelosis

Small artery

$15(3.5 \%) \quad 6(2.7 \%) \quad 9(4.3 \%)$

occlusion

Other mechanisms

$56(13.1 \%)$

$17(7.7 \%)$

$39(18.8 \%)$

NIHSS score at

13

15

$11<0.0001$

admission, median (IQR)

(9-19)

(10-21)

(8-16)

Stroke onset to

135

131

140

0.30

treatment time, minutes

(110-158)

(110-155)

(110-160)

IV antihypertensives

$80(18.6 \%)$

$37(16.7 \%)$

$43(20.7 \%)$

0.25

immediately prior to

rtPA

$\begin{array}{lllll}\text { Systolic blood pressure, } \quad 159.7 \pm 31.2 & 158.8 \pm 26.5 & 160.7 \pm 35.1 & 0.63\end{array}$ $\mathrm{mmHg}$

Diastolic blood pressure, $\quad 87.7 \pm 20.6 \quad 83.5 \pm 16.1 \quad 91.6 \pm 23.4 \quad 0.002$ $\mathrm{mmHg}$

Pulse rate, beat/minutes $\quad 80.8 \pm 19.4 \quad 79.3 \pm 18.1 \quad 82.3 \pm 20.6 \quad 0.24$ 
Table 2. Patient Imaging Findings

\begin{tabular}{|c|c|c|c|c|}
\hline Findings & $\begin{array}{c}\text { All } \\
\text { patients } \\
(\mathrm{n}=429)\end{array}$ & $\begin{array}{l}\text { Patients } \\
\text { over } 75 \\
\text { years old } \\
(\mathrm{n}=221)\end{array}$ & $\begin{array}{l}\text { Patients } \\
\text { under } 75 \\
\text { years old } \\
(\mathrm{n}=208)\end{array}$ & $\mathrm{p}$-value \\
\hline Occluded artery $(\mathrm{n}=341)$ & & & & 0.19 \\
\hline Middle cerebral artery & $\begin{array}{c}259 \\
(76.0 \%)\end{array}$ & $\begin{array}{c}142 \\
(77.2 \%)\end{array}$ & $\begin{array}{c}117 \\
(74.5 \%)\end{array}$ & \\
\hline Internal carotid artery & $\begin{array}{c}46 \\
(13.5 \%)\end{array}$ & $\begin{array}{c}25 \\
(13.6 \%)\end{array}$ & $\begin{array}{c}21 \\
(13.4 \%)\end{array}$ & \\
\hline $\begin{array}{l}\text { Anterior cerebral } \\
\text { artery }\end{array}$ & $\begin{array}{c}10 \\
(2.9 \%)\end{array}$ & $\begin{array}{c}7 \\
(3.8 \%)\end{array}$ & $\begin{array}{c}3 \\
(1.9 \%)\end{array}$ & \\
\hline Basilar artery & $\begin{array}{c}10 \\
(2.9 \%)\end{array}$ & $\begin{array}{c}3 \\
(1.6 \%)\end{array}$ & $\begin{array}{c}7 \\
(4.5 \%)\end{array}$ & \\
\hline $\begin{array}{l}\text { Posterior cerebral } \\
\text { artery }\end{array}$ & $\begin{array}{c}8 \\
(2.3 \%)\end{array}$ & $\begin{array}{c}4 \\
(2.2 \%)\end{array}$ & $\begin{array}{c}4 \\
(2.6 \%)\end{array}$ & \\
\hline Vertebral artery & $\begin{array}{c}8 \\
(2.3 \%)\end{array}$ & $\begin{array}{c}3 \\
(1.6 \%)\end{array}$ & $\begin{array}{c}5 \\
(3.2 \%)\end{array}$ & \\
\hline Occluded artery & 157 & 79 & 78 & 0.34 \\
\hline recanalization $(\mathrm{n}=260)$ & $(60.4 \%)$ & $(57.7 \%)$ & $(63.4 \%)$ & \\
\hline $\begin{array}{l}\text { ICH within the initial } 36 \\
\text { hours }\end{array}$ & $\begin{array}{c}86 \\
(20.2 \%)\end{array}$ & $\begin{array}{c}52 \\
(23.7 \%)\end{array}$ & $\begin{array}{c}34 \\
(16.5 \%)\end{array}$ & 0.06 \\
\hline
\end{tabular}


White matter lesions

$(\mathrm{WMLs})(\mathrm{n}=358)$

Grade 0

Grade 1

Grade 2

Grade 3
115

41

(32.1\%)

140

(39.1\%)

66

$(18.4 \%)$

37

$(10.3 \%)$
$(22.3 \%)$

66

(35.9\%)

48

$(26.1 \%)$

29

(15.7\%)
74

$(42.5 \%)$

74

$(42.5 \%)$

18

$(10.3 \%)$

8

$(4.7 \%)$ 
Table 3. Multivariate Logistic Regression Analysis of Factors Associated with ICH within the Initial 36 Hours

\begin{tabular}{lccc}
\hline & OR & $95 \% \mathrm{CI}$ & $\mathrm{p}$-value \\
\hline Over 75 years old & 1.18 & $0.57-2.47$ & 0.66 \\
Atrial fibrillation & 5.70 & $1.93-20.44$ & 0.001 \\
Previous stroke & 0.94 & $0.40-2.08$ & 0.87 \\
Cardioembolism & 1.39 & $0.39-5.12$ & 0.61 \\
NIHSS score at admission, & 1.06 & $1.01-1.11$ & 0.03 \\
per 1-point increase & & & $<0.0001$ \\
Recanalization & 4.95 & $2.23-12.29$ & \\
\hline
\end{tabular}


Table 4. Multivariate Logistic Regression Analysis of Factors Associated with an $\mathrm{m}-\mathrm{RS}$ score $>2$ at 3 months

\begin{tabular}{lccc}
\hline & OR & $95 \% \mathrm{CI}$ & $\mathrm{p}$-value \\
\hline Over 75 years old & 2.58 & $0.82-8.54$ & 0.11 \\
Hypertension & 4.14 & $1.32-14.79$ & 0.01 \\
NIHSS score at admission, & 1.08 & $0.99-1.19$ & 0.06 \\
per 1-point increase & & & \\
IV antihypertensives & 0.81 & $0.17-3.79$ & 0.79 \\
immediately prior to rtPA & & & \\
ICH within the initial 36 hours & 2.68 & $0.58-12.89$ & 0.20 \\
Internal carotid artery occlusion & 2.66 & $0.58-13.97$ & 0.21 \\
No recanalization & 10.10 & $3.03-39.33$ & 0.0001 \\
\hline
\end{tabular}


Fig 1

\section{$\square 0 \square 1 \square 2 \square 3 \square 4 \square 5 \square 6$}

Patients over 75-years

All patients $(n=191)$

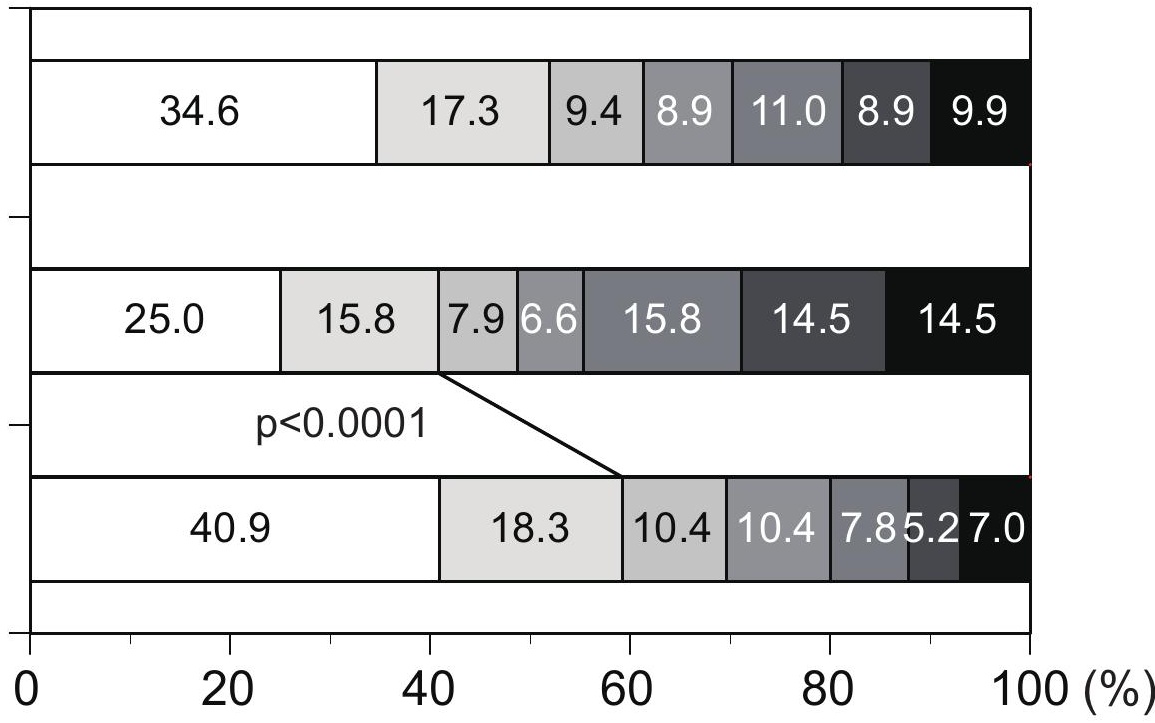

Patients under 75-years $(n=115)$ $(n=76)$ 